\title{
Ultrafast Magnetization Dynamics Studies using an X-ray Streak Camera
}

\author{
A. F. Bartelt, A. Comin, J. Feng, J. Nasiatka, H.A. Padmore, A. Scholl, A. Young \\ Advanced Light Source, Lawrence Berkeley National Laboratory, Berkeley, CA 94720, USA
}

\begin{abstract}
The spin dynamics of ferromagnetic thin films following an excitation by ultrashort 100-fs near-infrared laser pulses has recently received much attention. Here, a new approach is described using x-ray magnetic circular dichroism to investigate demagnetization and magnetization switching processes. In contrast to magneto-optical measurements, x-ray dichroism has the advantage of determining separately the spin and orbital components of the magnetic moment. The relatively low time resolution of the synchrotron x-ray probe pulses ( $80 \mathrm{ps} \mathrm{FWHM)}$ is overcome by employing an ultrafast $\mathrm{x}$-ray streak camera with a time resolution of $<1 \mathrm{ps}$. A description of the experimental setup including the $\mathrm{x}$-ray/IR laser pulse synchronization and the streak camera is given.
\end{abstract}

Keywords: Ultrafast magnetization dynamics, XMCD, x-ray streak camera

\section{INTRODUCTION}

The speed of magnetic recording is ultimately limited by the transition time between two states of different magnetization. In the quest for faster and more efficient magnetic data storage, non-conventional writing using either intense optical pulses or intense current pulses has captured much interest since it avoids a fundamental problem of magnetic fields: their slow decay with distance from the source. The long range of magnetic fields causes magnetic cross-talk in nano-scale devices and leads to inefficient use of the energy stored in the write field, since only a small fraction is localized near the structure that is to be switched. Experimentally it has been shown that optical pulses can manipulate the state of a magnet on a femtosecond time scale, three orders of magnitude faster than current storage technologies, leaving plenty of room for further improvement.

Vaterlaus et al. ${ }^{1}$ carried out first experiments studying the picosecond demagnetization of a ferromagnet by illuminating the sample with picosecond laser pulses. Later, Beaurepaire et al. ${ }^{2}$ and Scholl et al. ${ }^{3}$ showed that a faster demagnetization can be achieved using femtosecond laser pulses. Despite of the progress in femtosecond laser technology the origin of demagnetization is still controversial because optical techniques, such as the Magneto-Optical Kerr Effect (MOKE), are susceptible to bleaching as the carriers in the valence band are redistributed following the intense optical heating pulse. ${ }^{4,5}$ This lack of understanding of demagnetization is connected to a more general problem in magnetism: The lack of understanding of the nature of magnetic relaxation and magnetic damping. Damping is the result of magnetic friction which leads to energy loss and angular momentum transfer from the spin system to the lattice. In transformers, damping is a major source of energy waste, when the magnet state in the yoke is switched by an alternating current. Both magnetic damping and demagnetization have their roots in the spin-orbit interaction, which couples spin and orbital degrees of freedom.

X-ray techniques, such as x-ray magnetic circular dichroism (XMCD), have the unique ability to independently measure orbital and spin magnetization, quantitatively, and with element specificity. Time-resolved application of this technique in principle allows us to follow the transfer of angular momentum from spin to orbit to the lattice. X-ray probing is particularly well suited for the study of the complex magnetic materials typically used in recording: alloys, magnetic multilayers, and ferri- and antiferromagnets

Application of x-rays to the study of ultrafast processes has been slow because of the unavailability of ultrafast x-ray sources, in particular, sources which are suitable for spectroscopic studies. Laser-derived sources,

Further author information:

A.F.B.: E-mail: afbartelt@lbl.gov, Telephone: 15104866181

A.S.: E-mail: a_scholl@lbl.gov, Telephone: 15104864867 


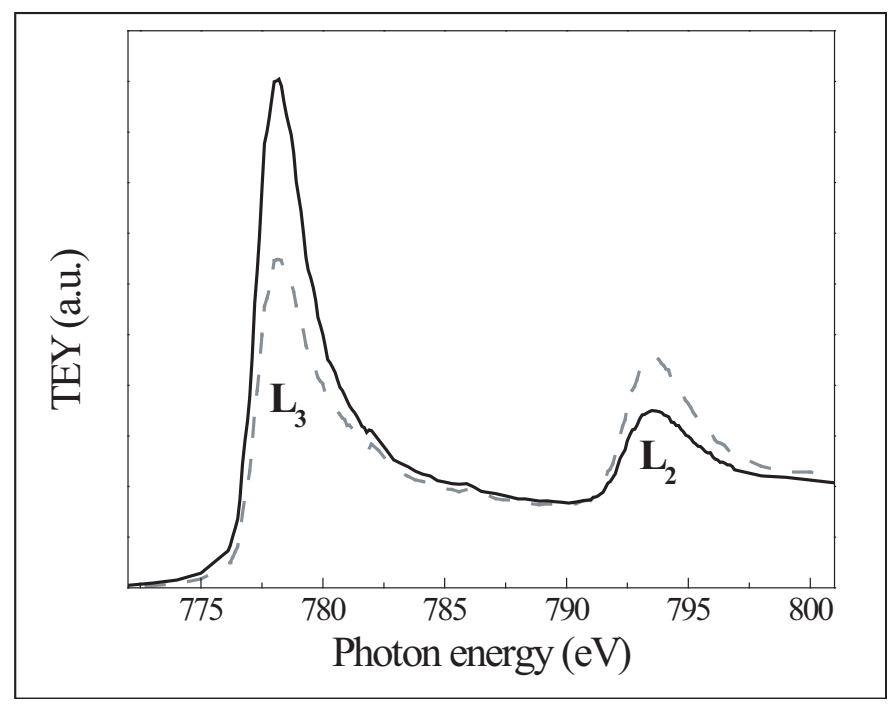

Figure 1. X-ray magnetic circular dichroism of Co measured using circularly polarized x-rays parallel (solid line) and antiparallel (dotted line) to the magnetization direction.

e.g., using high-harmonic generation ${ }^{6}$ or plasma techniques, ${ }^{7}$ have low repetition rates, low flexibility in tuning the energy, and are only starting to expand into the for spectroscopy important soft-x-ray region around 1 $\mathrm{keV}$. Currently, the premier source of x-rays are electron storage rings, which are pulsed sources of synchrotron radiation with repetition rates typically of hundreds of $\mathrm{MHz}$ and pulses lengths of about 100 ps. Electron storage rings produce x-rays either by deflecting a relativistic electron beam (usually several $\mathrm{GeV}$ ) using bending magnets or, at third generation rings, using mainly insertion devices. Latter are multipole magnet structures, in which the generated synchrotron radiation is either incoherently (wiggler) or partially coherently (undulator) superimposed. Synchrotron radiation from specialized, elliptically polarizing undulators (EPU) is polarized, and the polarization can be easily modified between linear and right and left circular by physically moving the magnet structure. ${ }^{8}$ Polarized x-rays have found application in studying anisotropic and magnetic systems using x-ray dichroism effects, which appear as a change in the x-ray absorption of the material depending on the angle between the x-ray polarization and the magnetization or anisotropy direction, Fig. 1. In magnetic materials x-ray sum rules were found that relate the size of the intensity change directly to the atomic magnetic moment. $^{9}$ X-ray magnetic circular dichroism (XMCD) is used today as an extremely versatile tool to study magnetism of films, surfaces, and interfaces with nanoscale spatial resolution, sub-monolayer sensitivity, and element-specificity. ${ }^{10}$ Element-specificity is particularly useful in alloys and multilayer systems. X-rays also give access to the magnetic properties of more complex magnetic systems such as ferrimagnets and antiferromagnets, which are studied with linearly polarized radiation using the x-ray magnetic linear dichroism effect (XMLD). XMCD and XMLD are both spectroscopic techniques and require x-rays tunable in energy and polarization. A high repetition rate, high flux, and low shot-to-shot variations in pulse intensity and beam position greatly benefit the experiments, improving the signal-to-noise and reducing the measurement time.

Two paths are currently being explored at third generation sources to improve the time resolution of timeresolved x-ray techniques, aiming for sub-picosecond time scale on which electronic and magnetic relaxation occur. In one approach ("Laser Slicing") a high-power laser pulse co-propagates with the electron bunch through a wiggler, thereby achieving a shift in energy of the sliced part of the bunch. The electron beam then travels through a bend magnet and a radiator, and x-rays originating from the sliced beam are separated and guided into an x-ray beamline. The slicing source promises to provide 100-200 femtosecond x-ray pulses at a repetition rate of tens of $\mathrm{kHz} .{ }^{11}$ Using an EPU, this method will potentially become an excellent source for time-resolved magnetic spectroscopy. A major advantage of laser slicing is the perfect temporal synchronization between the laser pump pulse and the laser-derived probe pulse. Disadvantages of laser slicing are the low flux per sliced 


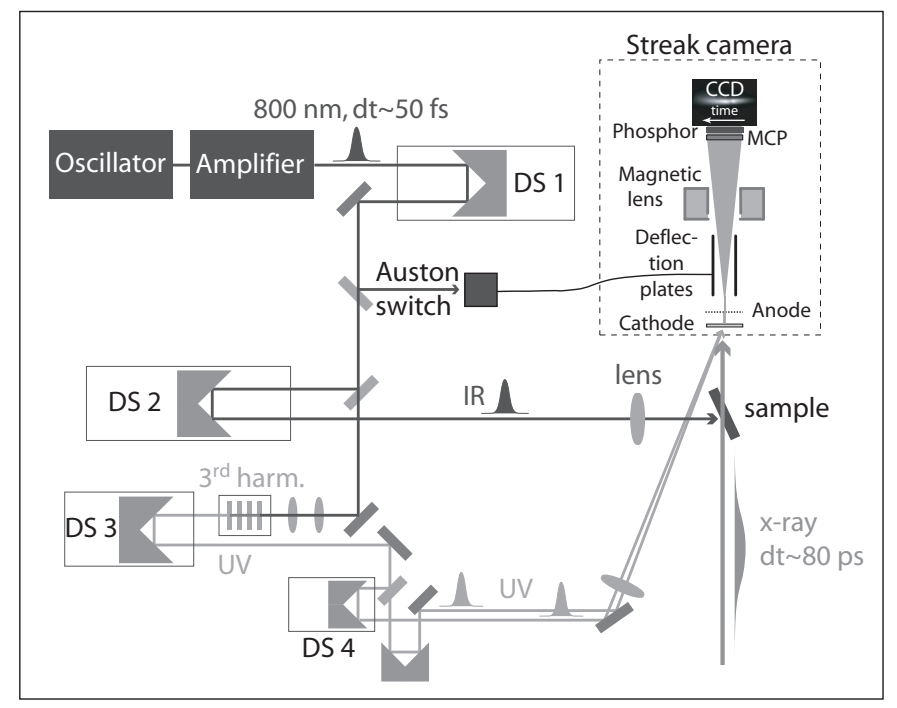

Figure 2. Experimental setup: Femtosecond IR pump pulses and 80 ps soft x-ray probe pulses coincide on the sample. The transmitted x-ray pulse is time-dispersed by the x-ray streak camera, which is triggered by an Auston switch. Femtosecond UV pulses are used for characterization and as time fiducial. All optical pulses are derived from the same 5 $\mathrm{kHz}$ laser amplifier, which is phase-locked to the x-ray pulses from the storage ring. A detailed description is given in the text.

$\mathrm{x}$-ray pulse, the relatively low repetition rate $(\mathrm{kHz})$ compared to the storage ring frequency $(\mathrm{MHz})$, and the requirement to sequentially measure a transient spectrum by changing the delay between the laser pump and the sliced x-ray probe. Also, laser-slicing experiments require a high initial investment and can only be done at a specialized beamline.

The disadvantages of laser slicing can partly be resolved by using an ultrafast streak camera detector in combination with a standard x-ray beamline. A time resolution of $280 \mathrm{fs}$ has been reported using UV illumination. ${ }^{12}$ Streak cameras can measure a full time spectrum in a single shot. They use the available x-ray flux more efficiently than laser slicing and in principle can be operated at RF frequencies, although currently with lower temporal resolution.

\section{SETUP AND RESULTS}

At the beamline 4.0.2 of the Advanced Light Source (ALS) we use an x-ray streak camera with a multi-shot time resolution of 0.9 ps using UV illumination (see contribution by J. Feng). The experimental setup is shown in Fig. 2. X-ray pulses from the synchrotron, which have a length of about $80 \mathrm{ps}$, pass through the sample and are detected on a $\mathrm{Au}$ photocathode. Secondary electrons from the cathode are accelerated in a static electric field to $10 \mathrm{kV}$ and are imaged by a magnetic solenoid onto a multi channel plate detector (MCP), which is read by a slow-scan charged-coupled device (CCD). The MCP is gated at the repetition rate of the laser amplifier, which is $5 \mathrm{kHz}$, to suppress the signal from the majority of the x-ray bunches. In multi-bunch operation, the usual operation mode of the ALS, a quasi-CW train of x-ray pulses, spaced by $2 \mathrm{~ns}$ is interrupted by a $100 \mathrm{~ns}$ gap. Inside the gap a single intense x-ray pulse ("cam-shaft") is located, whose current of initially $10 \mathrm{~mA}$ after a ring fill amounts to about $2.5 \%$ of the total ring current. The gap is asymmetrical with a larger gap before and a smaller gap after the cam-shaft pulse. This pattern is repeated at the round-trip frequency of the storage ring, which is $1.52 \mathrm{MHz}$. Fig. 3 shows that the gated channel plates suppress the intensity from the pulses before the cam-shaft gap to a large degree, however, fail to completely suppress intensity from pulses following the cam-shaft pulse. The remaining intensity is effectively suppressed by DC-biasing the deflection plates of the streak camera by $100-150 \mathrm{~V}$. 


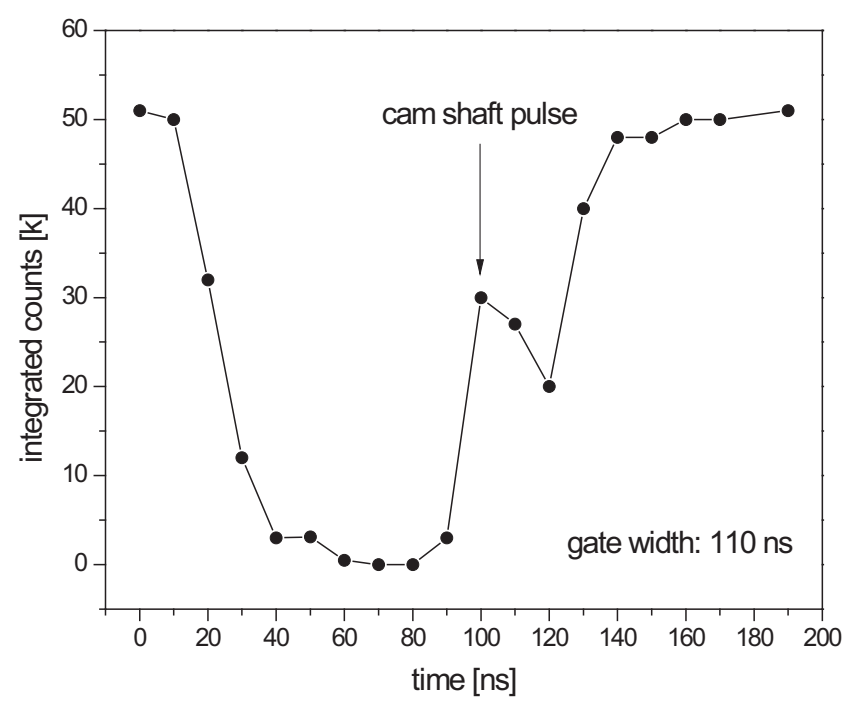

Figure 3. Streak intensity as function of time delay of the gating pulse on the multi-channel plates. The cam-shaft pulse resides in a $100 \mathrm{~ns}$ gap in the $\mathrm{x}$-ray pulse train.

The titanium-sapphire oscillator operates at $800 \mathrm{~nm}$ and a repetition rate of $62.5 \mathrm{MHz}, 1 / 8$ th of the repetition rate of the storage ring. It is phase-locked to the storage ring by dynamically adjusting the cavity length using a piezo-mounted mirror in the oscillator. The seed pulses from the oscillator are amplified by a $5 \mathrm{kHz}$ titaniumsapphire amplifier, which is synchronized to the round-trip frequency of the storage ring (1.52 MHz). The output of the amplifier is split into three beams, the first triggering a GaAs Auston switch, biased at about 200-300 V and driving the deflection plates of the streak camera, the second providing the pump beam, which is overlapped with the x-ray beam on the sample, and the third producing two UV pulses by frequency tripling to $266 \mathrm{~nm}$. The UV pulses are used for temporal characterization of the streak camera and will in the future provide a time fiducial to temporally align streaks, read out at the frequency of the laser. This will improve the multi-shot resolution of the streak camera, which is currently limited by the time jitter of the Auston switch. One of the challenges of the experiment is the loss of x-ray flux due to the low repetition rate of the laser. In multi-bunch mode only about $0.01 \%$ of the originally available $\mathrm{x}$-ray flux is used in an 80 -ps time window, determined by the length of the x-ray pulse. In a 1-ps time window the reduction in signal amounts to a factor of $10^{-6}$. An increase of the repetition rate of the streak camera, which is currently limited by the Auston switch and the available laser amplifier, would be extremely beneficial.

Sample rotation is possible and allows us to conduct measurements in grazing incidence for samples with an in-plane magnetization, and in normal incidence for samples with out-of-plane magnetization. Typical samples are thin layers ( $10 \mathrm{~nm}$ ) of magnetic material situated on a $200 \mathrm{~nm}$ thick $\mathrm{Al}$ heat sink, which provides thermal contact to the environment. Both are grown on a $100 \mathrm{~nm}$ thick $\mathrm{Si}_{3} \mathrm{~N}_{4}$ window with a size of $500 \times 500 \mu \mathrm{m}^{2}$. Sample heating above $600 \mathrm{~K}$ and cooling down to liquid $\mathrm{N}_{2}$ temperature are possible. A quadrupole magnet provides magnetic fields of up to $30 \mathrm{mT}$ along and perpendicular to the x-ray beam direction. All experiments are done in high vacuum $\left(10^{-7} \mathrm{mbar}\right)$, isolated from the ultra-high vacuum of the beamline by a thin $\mathrm{Al}$ window.

Fig. 4 shows a static x-ray pulse image measured using the streak camera. The exposure time was $15 \mathrm{~s}$, corresponding to an accumulation of $7.5 \cdot 10^{4}$ x-ray pulses. The streak shows an intensity profile commensurate with the approximately Gaussian temporal profile of the x-ray pulse. The structure in the image is due to defects in the phosphor of the MCP detector. This structure can be removed by flat-fielding the image. Below the x-ray streak an XMCD streak is shown. It was obtained from eight x-ray streak images measured on a Ni sample using opposite circular polarization, opposite magnetic fields of $\pm 20 \mathrm{mT}$, and for two photon energies corresponding to the $\mathrm{L}_{3}$ and $\mathrm{L}_{2}$ edge of Ni. The XMCD was calculated by subtracting the streak images of different polarizations and magnetic fields, and dividing the resulting streaks of the two energies. Ni is magnetic at room temperature 


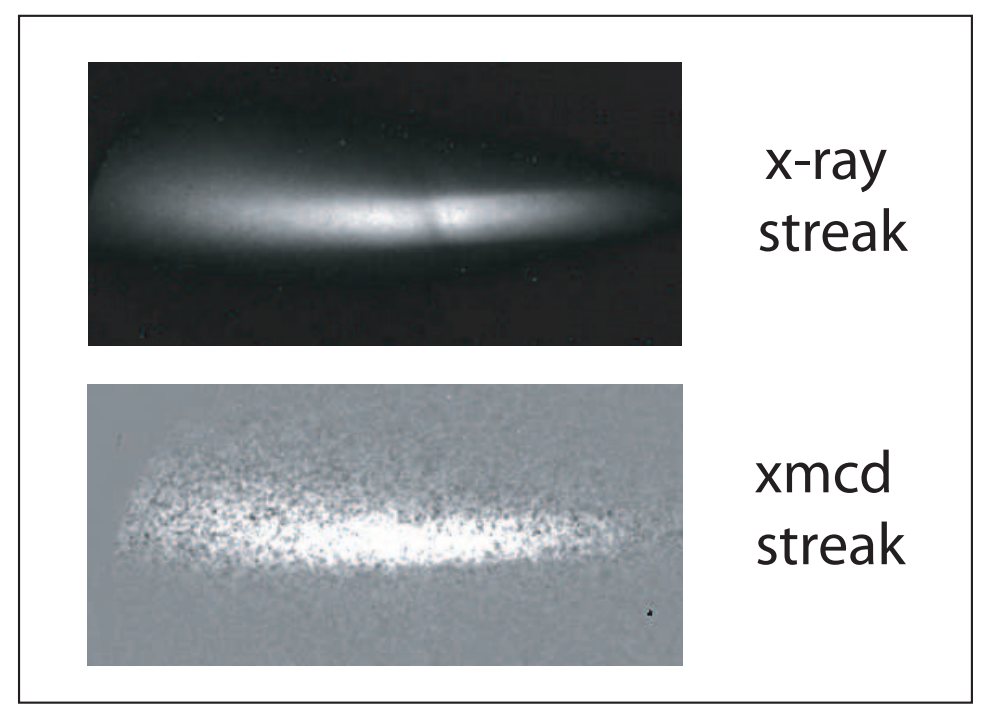

Figure 4. Top: 80 ps x-ray pulse measured using the streak camera. Bottom: Normalized difference between streaks measured with right and left circular polarization, opposite magnetization, and at the $\mathrm{L}_{3}$ and $\mathrm{L}_{2}$ edge of a Ni sample, demonstrating the magnetic sensitivity of the setup.

at which the measurement was performed. The Ni moment shows up as a bright contrast of the streak compared to the surrounding area. This measurement demonstrates the sensitivity of the streak camera to the magnetism of the sample. Measurements of the transient change of the magnetization of the sample after intense laser heating are currently being performed.

In summary, an x-ray streak camera has been installed at the Advanced Light Source for the study of fast transient magnetic processes in response to femtosecond laser heating. We anticipate a time resolution of about 1 ps using x-rays. A sub-picosecond time resolution has been achieved using UV illumination.

\section{ACKNOWLEDGMENTS}

This work was supported by the U.S. Department of Energy under Contract No. DE-AC02-05CH11231 and an LDRD at Lawrence Berkeley National Laboratory.

\section{REFERENCES}

1. A. Vaterlaus, D. Guarisco, M. Lutz, M. Aeschlimann, M. Stampanoni, and F. Meier, "Different spin and lattice temperatures observed by spin-polarized photoemission with picosecond laser pulses.," J. Appl. Phys. 67, p. 5661,1990 .

2. E. Beaurepaire, J.-C. Merle, A. Daunoisa, and J.-Y. Bigot, "Ultrafast spin dynamics in ferromagnetic nickel," Phys. Rev. Lett. 76, p. 4250, 1996.

3. A. Scholl, L. Baumgarten, R. Jacquemin, and W. Eberhardt, "Ultrafast spin dynamics of ferromagnetic thin films observed by fs spin-resolved two-photon photoemission," Phys. Rev. Lett. 79, p. 5146, 1997.

4. B. Koopmans, M. van Kampen, J. T. Kohlhepp, and W. J. M. de Jonge, "Ultrafast magneto-optics in nickel: magnetism or optics?," Phys. Rev. Lett. 85, p. 844, 2000.

5. M. van Kampen, C. Jozsa, J. T. Kohlhepp, P. LeClair, L. Lagae, W. J. M. de Jonge, and B. Koopmans, "All-optical probe of coherent spin waves," Phys. Rev. Lett. 88, p. 227201, 2002.

6. J. Seres, E. Seres, A. J. Verhoef, G. Tempea, C. Streli, P. Wobrauschek, V. Yakovlev, A. Scrinzi, C. Spielmann, and F. Krausz, "Laser technology: Source of coherent kiloelectronvolt x-rays," Nature 433, p. 596, 2005. 
7. C. Rose-Petruck, R. Jimenez, T. Guo, A. Cavalleri, C. W. Siders, F. Rksi, J. A. Squier, B. C. Walker, K. R. Wilson, and C. P. J. Barty, "Picosecond-milliangstrom lattice dynamics measured by ultrafast x-ray diffraction," Nature 398, p. 310, 1999.

8. A.T.Young, E. Arenholz, J. Feng, H. Padmore, S. Marks, R. Schlueter, E. Hoyer, N. Kelez, and C. Steier, "A soft x-ray undulator beamline at the advanced light source with circular and variable linear polarization for the spectroscopy and microscopy of magnetic materials," Surface Review and Letters 9, pp. 549-554, 2002.

9. B. T. Thole, C. Paolo, F. Sette, , and G. van der Laan, "X-ray circular dichroism as a probe of orbital magnetization," Phys. Rev. Lett. 68, p. 1943, 1992.

10. A. Scholl, H. Ohldag, F. Nolting, S. Anders, and J. Stoehr in Magnetic Microscopy of Nanostructures, H. Hopster, ed., Springer Verlag, 2004.

11. R. Schoenlein, S. Chattopadhyay, H. Chong, T. Glover, P. Heimann, C. Shank, A. Zholents, and M. Zolotorev, "Generation of femtosecond pulses of synchrotron radiation," Science 287, p. 2237, 2000.

12. M. Shakya and Z. Chang, "An accumulative x-ray streak camera with 280-fs resolution," in Proceedings of SPIE, H. Hopster, ed., 5534, p. 125, 2004. 